\title{
MODELOS DE EROSÃO HÍDRICA E TOLERÂNCIA DAS PERDAS DE SOLO EM LATOSSOLOS DISTRÓFICOS NO SUL DE MINAS GERAIS
}

\author{
André Silva Tavares $^{(\mathrm{a})}$, Henrique Mendes Junior ${ }^{(\mathrm{b})}$, Velibor Spalevic ${ }^{(\mathrm{c})}$, Ronaldo Luiz Mincato ${ }^{(\mathrm{d})}$ \\ (a) Mestrando em Ciências Ambientais, Universidade Federal de Alfenas-MG, E-mail: andresttavares@gmail.com \\ (b) Mestrando em Ciências Ambientais, Universidade Federal de Alfenas-MG, E-mail: mendesjr49@gmail.com \\ (c) Department of Geography, Faculty of Philosophy, University of Monte Negro, E-mail: \\ velibor.spalevic@gmail.com \\ (d) Instituto de Ciências da Natureza, Universidade Federal de Alfenas, E-mail: ronaldo.mincato@ unifal-mg.edu.br
}

\section{Eixo 12: Uso e Ocupação das Terras e Legislação Ambiental}

\begin{abstract}
Resumo
Diferentes métodos de cálculo das perdas de solo por erosão hídrica foram comparados com a Tolerância de Perda de Solo em Latossolos tropicais sob diferentes usos. Foram utilizados o Método de Erosão Potencial e a Equação Universal de Perda de Solo. O Método de Erosão Potencial foi calculado no aplicativo Intensidade de Erosão e Escoamento e obteve uma média de perdas de solo de $1,46 \mathrm{Mg} \mathrm{ha}^{-1}$ ano $^{-1}$ com descarga máxima de $649,31 \mathrm{Mg}$ ano $^{-1}$. Já, pela Equação Universal de Perda de Solo foi obtida uma média de perda de solo de $1,52 \mathrm{Mg} \mathrm{ha}^{-1} \mathrm{ano}^{-1}$, com descarga máxima de 668,26 $\mathrm{Mg}$ ano $^{-1}$. As perdas de solo calculadas pelos dois métodos foram similares e ficaram aquém do limite de Tolerância de Perda de Solo, que variou de 5,19 a 5,90 $\mathrm{Mg} \mathrm{ha}^{-1}$ ano ${ }^{-1}$ e validam a aplicação do Método da Erosão Potencial em solos tropicais do sul de Minas Gerais.
\end{abstract}

Palavras chave: EPM - RUSLE - IntErO - degradação do solo.

\section{Introdução}

O processo de aceleração da erosão hídrica por ações antrópicas causa perdas de solo, água e nutrientes essenciais para o equilíbrio sustentável da agropecuária (PANAGOS et al., 2015). As taxas de perdas de solo, no Brasil variam de 15 a $25 \mathrm{Mg} \mathrm{ha}^{-1}$ ano $^{-1}$, enquanto que as de formação do solo estão em cerca de 1 $\mathrm{Mg} \mathrm{ha}^{-1}$ ano $^{-1}$ (BERTONI; LOMBARDI NETO, 2012; PIMENTEL et al., 1995).

Métodos empíricos como a Revised Universal Soil Loss Equation, RUSLE (RENARD et al., 1997) e físicos como o Erosion Potential Method, EPM (Gravilovic, 1988), foram desenvolvidos para modelar as perdas de solo por erosão hídrica a partir de técnicas de geoprocessamento, imagens de satélites e atributos físicos e químicos do solo.

A modelagem das perdas de solo permite o conhecimento das áreas com maior intensidade de erosão e comparar com o limite da Tolerância de Perda de Solo (TPS). A TPS representa a perda de solo máxima tolerável para manter a capacidade produtiva do solo e no Brasil é calculada, principalmente, pelo método 
de Bertol e Almeida (2000), por considerar no cálculo um maior número de atributos do solo de fácil obtenção (CÂNDIDO et al., 2014; OLIVETTI et al., 2015).

A região do Sul de Minas Gerais possui grandes áreas de cultivo do café, porém com carência de avaliações das perdas de solo. Carvalho et al. (2007) calcularam as perdas de solo em Latossolos da região com parcelas padrões pelo método da Equação Universal de Perdas de Solos (Universal Soil Loss Equation - USLE) (WISHMEIER; SMITH, 1978) e verificaram perdas de solos menores nos sistemas de manejo mais adensado em comparação aos mais espaçados. Portanto, a modelagem da erosão hídrica nessa área permite quantificar as perdas de solo e comparar à TPS, visando avaliar e orientar as práticas de manejo necessárias para a sustentabilidade no uso do solo.

Assim, neste trabalho foram calculadas as perdas de solo por erosão hídrica pelos modelos RUSLE e EPM e comparadas com os limites da TPS em Latossolos sob diferentes usos.

\section{Materiais de Métodos}

A área de estudo compreende a Sub-bacia Hidrográfica do Córrego da Laje, com área de 437 ha, situada na Fazenda Capoeirinha da Ipanema Agrícola S.A. (Ipanema Coffees), nas coordenadas $21^{\circ} 29^{\prime}$ a $21^{\circ} 33^{\prime}$ $\mathrm{S}$ e $45^{\circ} 52^{\prime}$ a $45^{\circ} 59^{\prime} \mathrm{W}$. A Sub-bacia do Córrego da Laje possui perímetro de 9,90 km e altitudes entre 814 e 914 metros e é afluente direta do Reservatório da Usina Hidrelétrica de Furnas, na Bacia Hidrográfica do Rio Grande, Sul de Minas Gerais.

O clima, na classificação de Köppen, é o Tropical Mesotérmico (CwB), com precipitação média de 1.500 mm ano $^{-1}$ (SPAROVEK; VAN LIER; DOURADO NETO, 2009). O relevo pertence à unidade fisiográfica dos mares de morros, na formação da Unidade litológica Paragnáissica Migmatítica Superior VarginhaGuaxupé (HASUI, 2010).

As classes de uso do solo da sub-bacia são: café (237,35 ha); milho e feijão com cultivo em sucessão (60,49 ha); eucalipto (16,95 ha); área de preservação permanente (APP) (90,59 ha); carreadores (divisores de glebas) (9,71 ha); usina de adubo (2,40 ha) e drenagens (19,51 ha). As APP equivalem a 20,73\% da sub-bacia e estão de acordo com o Código Florestal Brasileiro (BRASIL, 2012) respeitando os $50 \mathrm{~m}$ para o entorno das nascentes e os $30 \mathrm{~m}$ para margens dos cursos d'água. A área de mata nativa considerada nos cálculos das perdas de solo foi de 68,99 ha.

O Modelo Digital de Elevação (MDE), com resolução (pixels) de 10 metros (Figura 1), foi obtido a partir da interpolação das curvas de nível da Carta Topográfica do Município de Alfenas (FOLHA SF 23-1-1-3) na escala de 1: 50.000 (IBGE, 1970) pela ferramenta topo to raster do software ArcGis 10.3. 


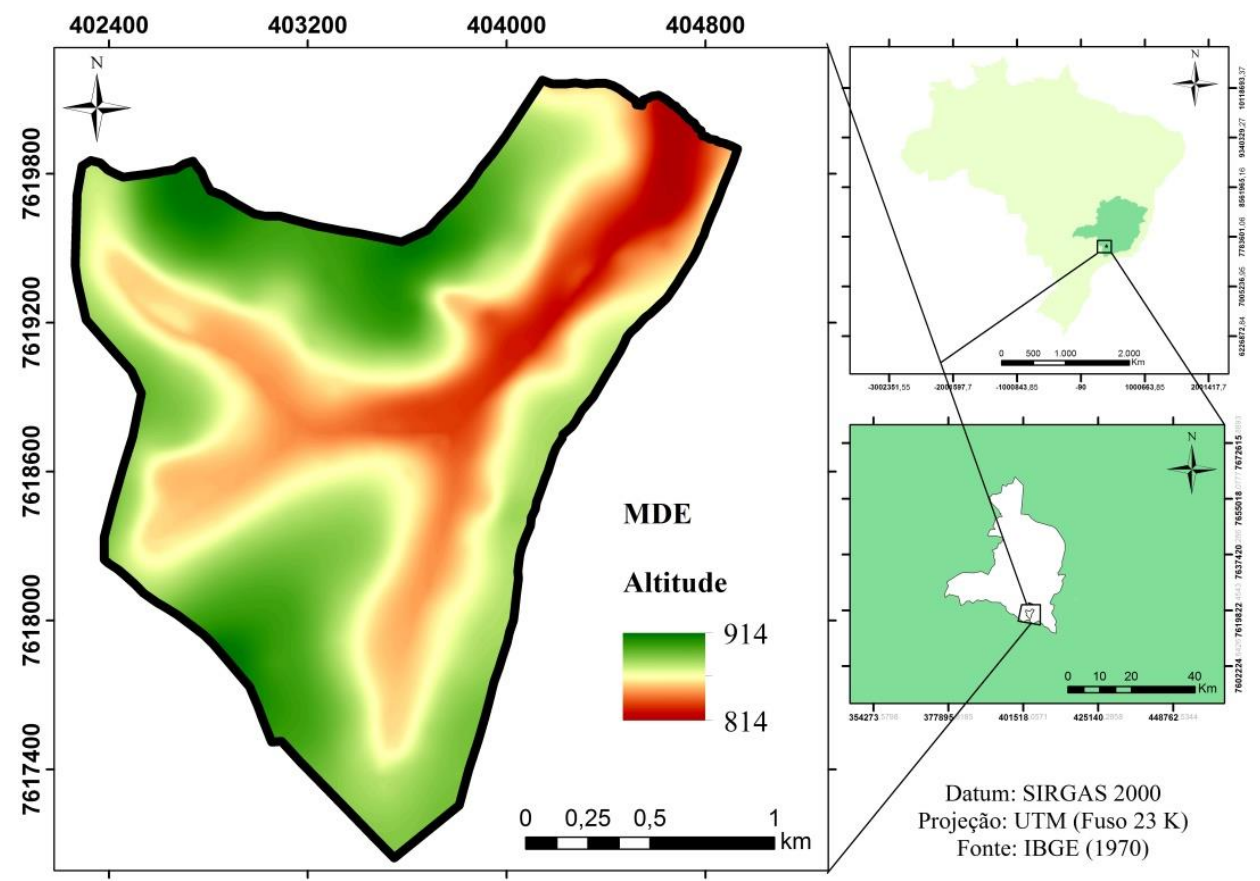

Figura 1 - Mapa de Localização e Modelo Digital de Elevação da Sub-bacia Hidrográfica do Córrego da Laje.

A partir do MDE foi confeccionado o mapa de declividade, por meio da ferramenta Slope do ArcGis 10.3, que serviu como base para a obtenção das classes de solo, conforme o modelo Scorpan de Mcbratney et al. (2003). O mapa digital de solos foi elaborado com base nas características do solo. Para tanto foram coletadas 108 amostras: entre deformadas, indeformadas em cilindro $\left(92,53 \mathrm{~cm}^{3}\right)$ e indeformadas em forma de torrão.

As análises de solo realizadas nos laboratórios do Departamento de Ciência do Solo da Universidade Federal de Lavras - UFLA. A descrição morfológica foi realizada no campo em micro trincheiras (40 x $40 \times 60 \mathrm{~cm}$ ), conforme os procedimentos de Santos et al. (2005). O mapa digital de solos (Figura 2A) em nível semidetalhado (IBGE, 2015) considerou as classes de relevo da Embrapa (2013) (Figura 2B). Os solos foram classificados em Latossolo Vermelho distrófico em relevo plano a suave ondulado (LVd1), ondulado (LVd2), forte ondulado ( $\mathrm{LVd} 3)$ e solos indiscriminados de várzea (SIV) nas zonas de deposição.

- Perdas de solo no modelo RUSLE: a perda média de solo pelo modelo RUSLE (RENARD et al., 1997) é baseada na equação 1 .

$$
\mathrm{A}=\mathrm{R} * \mathrm{~K} * \mathrm{LS} * \mathrm{C} * \mathrm{P} \quad \text { (equação 1) }
$$




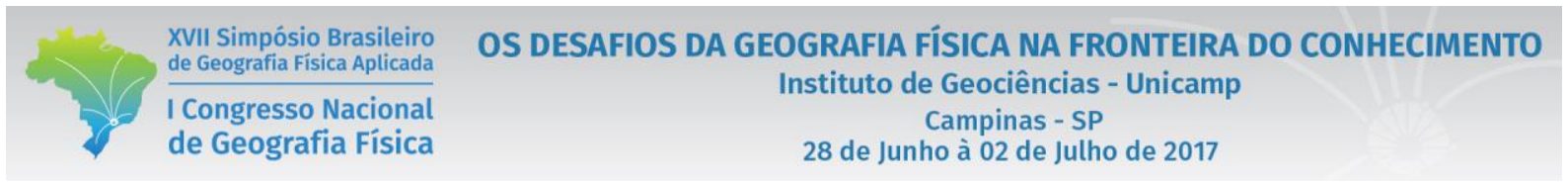

Em que: $\mathrm{A}=$ perda de solo média anual $\left(\mathrm{Mg} \mathrm{ha}^{-1} \mathrm{ano}^{-1}\right) ; \mathrm{R}=$ erosividade da chuva $\left(\mathrm{MJ} \mathrm{mm} \mathrm{ha-1} \mathrm{h}^{-1}\right.$ ano $\left.^{-1}\right)$; $\mathrm{K}=$ erodibilidade do solo $\left(\mathrm{Mg} \mathrm{h} \mathrm{MJ}^{-1} \mathrm{~mm}^{-1}\right) ; \mathrm{LS}=$ fator topográfico, que envolve o comprimento e a declividade da rampa (adimensional); $\mathrm{C}=$ uso e manejo do solo (adimensional); $\mathrm{P}=$ práticas conservacionistas no solo (adimensional).
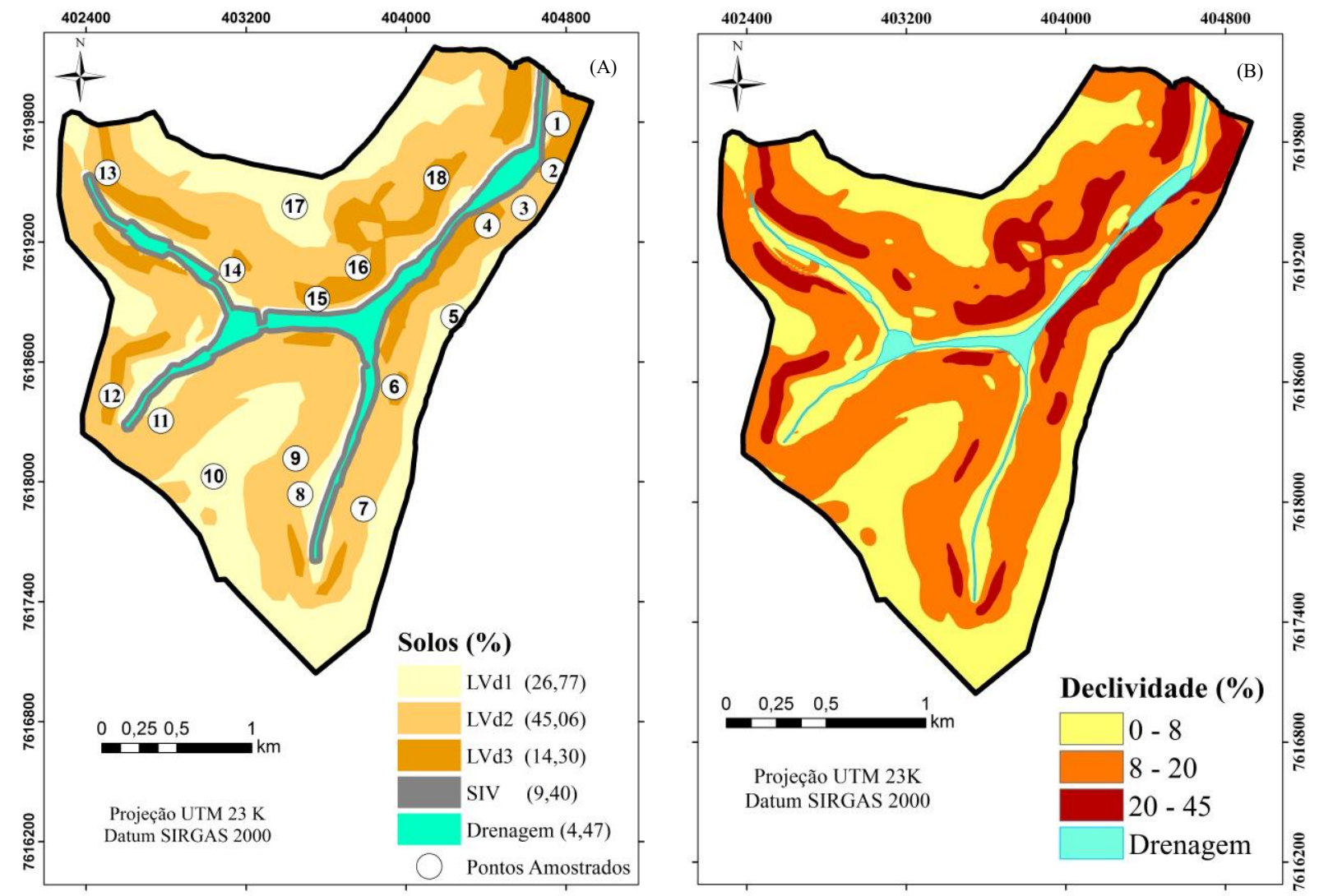

Figura 2: (A) Mapa Digital de Solos em área percentual e (B) Mapa de Declividade da Sub-bacia hidrográfica do Córrego da Laje, Alfenas, sul de Minas Gerais. Latossolo Vermelho Distrofico em relevo plano a suave ondulado (LVd1), ondulado (LVd2) e forte ondulado (LVd3). Solos Indiscriminados de Varzea (SIV).

A erosividade da chuva (R) foi obtida de Aquino et al. (2012). A erodibilidade do solo (K) foi calculada com base nas descrições morfológicas e analise física das amostras coletadas sob mata nativa, utilizando o método indireto número 1 de Silva et al. (1999), expresso na equação 2.

$Y=4,77 \times 10^{-2}-9,66 \times 10^{-3} X_{14}+1,63 \times 10^{-2} X_{16}-1,12 \times 10^{-2} X_{17}+1,85 \times 10^{-2} X_{18}-1,51 \times 10^{-2} X_{19}-2,46$ x $10^{-4} \mathrm{X}_{22}-3,58 \times 10^{-4} \mathrm{X}_{23}+1,47 \times 10^{-4} \mathrm{X}_{24}-1,43 \times 10^{-4} \mathrm{X}_{25}+3,26 \times 10^{-3} \mathrm{X}_{26}-1,26 \times 10^{-3} \mathrm{X}_{27}-2,29 \times 10^{-}$ ${ }^{4} \mathrm{X}_{31}+1,07 \times 10^{-4} \mathrm{X}_{32}+2,69 \times 10^{-4} \mathrm{X}_{34}$ (equação 2)

Em que: $\mathrm{Y}=$ erodibilidade em $\mathrm{Mg} \mathrm{h} \mathrm{MJ}^{-1} \mathrm{~mm}^{-1} ; \mathrm{X}_{14}$ = código do matiz do solo úmido segundo Munsell (adimensional); $\mathrm{X}_{16}=$ código do grau da estrutura (adimensional); $\mathrm{X}_{17}=$ código do tamanho da estrutura (adimensional); $\mathrm{X}_{18}=$ código da forma da estrutura (adimensional); $\mathrm{X}_{19}=$ código da plasticidade do solo 
(adimensional); $\mathrm{X}_{22}=$ teor de areia fina dispersa com NaOH $0,1 \mathrm{~mol} \mathrm{~L}^{-1}\left(\mathrm{~g} \mathrm{~kg}^{-1}\right) ; \mathrm{X}_{23}=$ teor de areia muito fina dispersa com $\mathrm{NaOH} 0,1 \mathrm{~mol} \mathrm{~L}^{-1}\left(\mathrm{~g} \mathrm{~kg}^{-1}\right) ; \mathrm{X}_{24}=$ teor de silte disperso com $\mathrm{NaOH} 0,1 \mathrm{~mol} \mathrm{~L}^{-1}\left(\mathrm{~g} \mathrm{~kg}^{-1}\right)$; $\mathrm{X}_{25}=$ teor de argila dispersa com $\mathrm{NaOH} 0,1 \mathrm{~mol} \mathrm{~L}^{-1}\left(\mathrm{~g} \mathrm{~kg}^{-1}\right) ; \mathrm{X}_{26}=$ teor de areia muito grossa disperso em água $\left(\mathrm{g} \mathrm{kg}^{-1}\right) ; \mathrm{X}_{27}=$ teor de areia grossa disperso em água $\left(\mathrm{g} \mathrm{kg}^{-1}\right) ; \mathrm{X}_{31}=$ teor de silte disperso em água $(\mathrm{g}$ $\left.\mathrm{kg}^{-1}\right) ; \mathrm{X}_{32}=$ teor de argila dispersa em água $\left(\mathrm{g} \mathrm{kg}^{-1}\right)$ e $\mathrm{X}_{34}=$ índice de floculação (adimensional).

As variáveis X14, X16, X17, X18 e X19 foram obtidas pela descrição morfológica do solo em campo. As variáveis X22, X23, X24, X25, X26, X27, X31 e X32 foram determinadas em laboratório pela análise granulométrica do solo. A variável X34 foi obtida de Embrapa (2011).

A topografia (LS) foi determinada pelo modelo $\mathrm{LS}_{\mathrm{RUSLE}}$ 3D de Mitasova et al. (1996), representado pela equação 3.

$$
\mathrm{LS}_{\text {RUSLE 3D }}=(\mathrm{m}+1)(\mathrm{A} / 22,13)^{\mathrm{m}}(\operatorname{sen} \Theta / 0,09)^{\mathrm{n}} \quad(\text { equação 3) }
$$

Em que: $\mathrm{LS}_{\mathrm{RUSLE}} 3 \mathrm{D}=$ fator topográfico (adimensional); $\mathrm{A}=$ área de contribuição a montante por unidade de comprimento de célula para um $\operatorname{MDE}\left(\mathrm{m}^{2}\right) ; \Theta=$ ângulo de inclinação do declive (graus) e $\mathrm{m}$ e $\mathrm{n}=$ parâmetros empíricos com variação do m entre 0,4 a 0,6 e do n entre 1,0 a 1,4 em função do tipo de erosão predominante (laminar ou em sulcos).

Os fatores de uso e manejo do solo (C) e práticas conservacionistas (P) foram obtidos da literatura especializada para cada uso do solo e do mapa das classes de uso do solo.

$\mathrm{O}$ fator $\mathrm{P}$ foi determinado com base nas práticas conservacionistas adotadas na área e o mapa das classes de uso do solo. O cultivo do café é realizado em curvas de nível com plantio em contorno e o milho e o feijão com plantio direto. Nos povoamentos de eucalipto não foi verificada nenhuma prática conservacionista e foi caracterizado como plantio morro abaixo. Para mata nativa foi atribuído valor zero por ser área de preservação.

- Perdas de solo no modelo EPM: A perda de solo $\left(\mathrm{W}_{\mathrm{yr}}\right)$ no modelo EPM é estimada pela equação 4 (GAVRILOVIC, 1988). Os seus algoritmos estão incorporado no aplicativo IntErO (SPALEVIC, 2011), que calcula de forma automatizada, evitando erros na modelagem manual.

$$
\mathrm{W}_{\mathrm{yr}}=\mathrm{T} \cdot \mathrm{H}_{\mathrm{yr}} \cdot \pi \cdot \sqrt{ } \mathrm{Z}^{3} \cdot \mathrm{F} \quad(\text { equação } 4)
$$

Em que: $\mathrm{W}_{\mathrm{yr}}=$ produção total de sedimentos $\left(\mathrm{m}^{3}\right.$ ano $\left.^{-1}\right) ; \mathrm{T}=$ coeficiente de temperatura (adimensional); $\mathrm{H}_{\mathrm{yr}}=$ precipitação média $\left(\mathrm{mm}\right.$ ano $\left.^{-1}\right) ; \pi=3,14 ; \mathrm{Z}=$ coeficientes de erosão (adimensional); $\mathrm{F}=$ área da bacia hidrográfica $\left(\mathrm{km}^{2}\right)$.

O coeficiente de temperatura (T) é calculado conforme a equação 5. 
XVII Simpósio Brasileiro

de Geografia Física Aplicada

I Congresso Nacional

de Geografia Física
OS DESAFIOS DA GEOGRAFIA FÍSICA NA FRONTEIRA DO CONHECIMENTO

Instituto de Geociências - Unicamp

Campinas - SP

28 de Junho à 02 de Julho de 2017

$$
\left.\mathrm{T}=\sqrt{ }\left(\mathrm{t}_{0} / 10\right)+0,1 \quad \text { (equação } 5\right)
$$

Em que: $\mathrm{T}=$ coeficiente de temperatura (adimensional); $\mathrm{t}_{0}=$ temperatura média do $\operatorname{ar}\left({ }^{\circ} \mathrm{C}\right.$ ano $\left.{ }^{-1}\right)$.

A temperatura média do ar $\left(\mathrm{t}_{0}\right)$ é de $22^{\circ} \mathrm{C}$, com máximas variando de 28 a $30^{\circ} \mathrm{C}$ nos períodos mais secos e no inverno as médias mensais variam 17 a $19^{\circ} \mathrm{C}$.

O coeficiente de erosão $(Z)$ é obtido pela equação 6:

$$
\mathrm{Z}=\mathrm{Y} \cdot \mathrm{X}_{\mathrm{a}} \cdot\left(\varphi+\mathrm{I}_{\mathrm{sr}}\right) \quad \text { (equação 6) }
$$

Em que: $\mathrm{Y}=$ resistência do solo a erosão hídrica (adimensional); $\mathrm{X}_{\mathrm{a}}=$ uso e manejo do solo (adimensional); $\varphi$ = grau de erosão no terreno (adimensional); $\mathrm{I}_{\mathrm{sr}}=$ inclinação média da sub-bacia (\%).

Gavrilovic (1988) preparou tabelas com valores que representam os atributos $\left(\mathrm{Y}, \mathrm{X}_{\mathrm{a}}, \varphi\right)$ necessários para o cálculo do coeficiente de erosão (Z). Entretanto, o modelo foi aplicado inicialmente em regiões de clima temperado, sendo necessário adaptar os valores de acordo com as características dos solos tropicais brasileiros.

O coeficiente de retenção de sedimentos $\left(R_{u}\right)$ é calculado pela equação 7 .

$$
\mathrm{R}_{\mathrm{u}}=(\mathrm{O} \cdot \mathrm{D})^{0,5} / 0,25\left(\mathrm{~L}_{\mathrm{v}}+10,0\right) \quad \text { (equação 7) }
$$

Em que: $\mathrm{O}=$ comprimento da sub-bacia $(\mathrm{km}) ; \mathrm{D}=$ diferença de elevação da sub-bacia $(\mathrm{m}) ; \mathrm{L}_{\mathrm{v}}=$ comprimento do córrego principal da sub-bacia $(\mathrm{km})$.

- Tolerância de Perda de Solo: a TPS foi obtida pelo método de Bertol e Almeida (2000) (equação 8).

$$
\mathrm{TPS}=\mathrm{h} \mathrm{r} \mathrm{r}_{\mathrm{a}} \mathrm{m} 1.000^{-1} \quad \text { (equação 8) }
$$

Em que: TPS = tolerância de perda de solo $\left(\mathrm{em} \mathrm{Mg} \mathrm{ha}^{-1} \mathrm{ano}^{-1}\right.$, corrigida da formula original considerando a densidade do solo; $\mathrm{h}=$ profundidade efetiva do solo $(\mathrm{mm})$, limitada a $1.000 \mathrm{~mm} ; \mathrm{r}_{\mathrm{a}}=$ relação que expressa, conjuntamente, o efeito da relação textural entre os horizontes B e A e do teor de argila do horizonte $\mathrm{A} ; \mathrm{m}=$ fator que expressa o efeito da matéria orgânica na camada de $0-20 \mathrm{~cm}$ do solo; $\mathrm{p}=$ fator que expressa o efeito da permeabilidade do solo e $1.000^{-1}=$ constante que expressa o período de tempo necessário para desgastar uma camada de solo de $1.000 \mathrm{~mm}$ de espessura.

A profundidade efetiva (variável h) para todas as unidades de mapeamento foi de $1.000 \mathrm{~mm}$ (BRASIL, 1962). Para a variável $r_{a}$ foi utilizado o teor de argila dispersa com $\mathrm{NaOH} 0,1 \mathrm{~mol} \mathrm{~L}-1$ que variou de 584 a $607 \mathrm{~g} \mathrm{~kg}-1$. Assim, primeiro, foi obtido a relação textural entre as camadas superficial $(0-20 \mathrm{~cm})$ e subsuperficial $(20-60 \mathrm{~cm}$ ) de cada amostragem (EMBRAPA, 2013). A variável $\mathrm{m}$ representa o teor médio de matéria orgânica na camada superficial $(0-20 \mathrm{~cm})$ de cada classe de solo. A variável permeabilidade do solo, p, foi obtida em campo, a partir de três repetições para cada classe de solo (ZHANG, 1997) com Infiltrômetro Mini Disk Decagon Devices ajustado para taxa de sucção de $2 \mathrm{~cm}$. A 


\section{OS DESAFIOS DA GEOGRAFIA FISICA NA FRONTEIRA DO CONHECIMENTO \\ Instituto de Geociências - Unicamp \\ Campinas - SP \\ 28 de Junho à 02 de Julho de 2017}

permeabilidade do solo foi classificação de acordo com Soil Survey Division Staff (1993) e Galindo e Margolis (1989) que utilizam a textura e o grau de estrutura do solo.

\section{Resultados e Discussões}

- Modelo RUSLE: o fator R para a área é de $6.500 \mathrm{MJ} \mathrm{mm} \mathrm{ha}^{-1} \mathrm{~h}^{-1} \mathrm{ano}^{-1}$ (AQUINO et al., 2012). O fator $\mathrm{K}$ foi de 0,021, 0,004 e 0,026 $\mathrm{Mg} \mathrm{h} \mathrm{MJ}^{-1} \mathrm{~mm}^{-1}$, respectivamente, para LVD1, LVD2 e LVD3. O fator LS variou de 0 a 3,883, com média de 0,318 . Os valores da literatura utilizados para os fatores $\mathrm{C}$ e $\mathrm{P}$ estão listados na tabela I.

Tabela I - Fatores C e P para os usos e manejos verificados na Sub-bacia Hidrográfica do Córrego da Laje.

\begin{tabular}{lccc}
\hline \multicolumn{1}{c}{ Uso e Manejo } & Fator C & Fator $\mathrm{P}^{\mathbf{1}}$ & Autor \\
\hline $\begin{array}{l}\text { Café com espaçamento de 3,0 x } \\
\text { 0,5 m e plantio em nível }\end{array}$ & 0,1354 & 0,50 & Prochnow et al. (2005) \\
$\begin{array}{l}\text { Milho + feijão com plantio } \\
\text { direto }\end{array}$ & 0,0271 & 0,01 & Bertol et al. (2001) \\
Eucalipto & & & \\
Mata nativa & 0,1240 & 1,00 & Silva et al. (2016) \\
Carreador (divisor de glebas) & 0,0150 & 0,00 & Silva et al. (2016) \\
\hline
\end{tabular}

Nota: ${ }^{1}$ Valores de P obtidos de Bertoni e Lombardi Neto (2012); Roose (1977).

A perda de solo média foi de $1,52 \mathrm{Mg} \mathrm{ha}^{-1}$ ano $^{-1}$ com variação de 0,01 a $18,77 \mathrm{Mg} \mathrm{ha}^{-1}$ ano ${ }^{-1}$. Os maiores valores estão concentrados nos carreadores do café, com 18,77 $\mathrm{Mg} \mathrm{ha}^{-1}$ ano $^{-1}$, que também possui os fatores C, P e LS elevados pelo fato de configurar solo exposto posicionados, na maioria, em terreno de declividade forte ondulada $(20-45 \%)$. Já os menores valores de perda do solo estão associados à mata nativa com $0,01 \mathrm{Mg} \mathrm{ha}^{-1}$ ano $^{-1}$. Os cultivos do milho e feijão em sucessão apresentaram perdas de solo média de $0,12 \mathrm{Mg} \mathrm{ha}^{-1} \mathrm{ano}^{-1}$, o eucalipto de 6,08 $\mathrm{Mg} \mathrm{ha}^{-1} \mathrm{ano}^{-1}$ e o café de 1,58 $\mathrm{Mg} \mathrm{ha}^{-1}$ ano $^{-1}$ (tabela II). Este valor para o café, foi abaixo do encontrado por Silva et al. (2007), de 10,98 $\mathrm{Mg} \mathrm{ha}^{-1}$ ano $^{-1}$ para Latossolo Vermelho-Amarelo Distrófico com café conilon em espaçamento 2,90 x 0,90 m cultivado há cinco anos em Cachoeiro de Itapemirim - ES. Essa diferença pode estar associada à distinção entre a erodibilidade dos solos, de $0,14 \mathrm{Mg} \mathrm{h} \mathrm{MJ}^{-1} \mathrm{~mm}^{-1}$ (Silva et al., 2007) e de 0,004 a $0,026 \mathrm{Mg} \mathrm{h} \mathrm{MJ}^{-1} \mathrm{~mm}^{-1}$ neste trabalho. 
- Modelo EPM: A precipitação média $\left(\mathrm{H}_{\mathrm{yr}}\right)$ é de $1.500 \mathrm{~mm} \mathrm{ano}^{-1}$ (SPAROVEK et al., 2007). O coeficiente de temperatura (T) obtido foi de 1,52. A diferença de elevação na sub-bacia (D), que representa a distância média percorrida pelo escoamento até o interflúvio, foi de 37,21 m. O perímetro (O) é de 9,90 $\mathrm{km}$ e o comprimento do córrego principal $\left(\mathrm{L}_{\mathrm{v}}\right)$ é de $2,98 \mathrm{~km}$, com largura média de $40 \mathrm{~m}$.

De acordo com o mapa de declividade, a inclinação media da sub-bacia ( $\left.\mathrm{I}_{\mathrm{sr}}\right)$ foi de 11,6 \%, o que indica um relevo com declividade média. A resistência à erosão hídrica $(\mathrm{Y})$ dos Latossolos Vermelhos distróficos foi calculada em 0,90, na escala de 0,25 a 2,0, que indica uma resistência moderada à erosão hídrica. A média do coeficiente para uso e manejo (Xa) foi de 0,55 , o que indica um bom manejo no uso do solo, isso devido às práticas de conservação nos plantios em nível e direto, em mais de $60 \%$ da área.

O valor médio para o grau de erosão no terreno $(\varphi)$ foi de 0,40 , o que sugere processos de erosão do tipo fraco considerando a área da sub-bacia. A intensidade média da erosão $(Z)$ foi de 0,37 , indicando que a sub-bacia é classificada na categoria IV de deterioração, ou seja, de erosão do tipo fraca. O coeficiente de retenção de sedimentos $\left(\mathrm{R}_{\mathrm{u}}\right)$ foi de 0,093 , ou seja, 9,3\% dos sedimentos produzidos no total das perdas de solo são transportados ao interflúvio.

A perda média de solo foi de 1,46 $\mathrm{Mg} \mathrm{ha}^{-1}$ ano $^{-1}$, variável de 0,10 a 5,50 $\mathrm{Mg} \mathrm{ha}^{-1} \mathrm{ano}^{-1}$. As áreas com maior perda estão concentradas nos carreadores de café, com 5,50 $\mathrm{Mg} \mathrm{ha}^{-1} \mathrm{ano}^{-1}$. Os maiores valores são devidos ao solo exposto nos terrenos com declividades forte onduladas $(20-45 \%)$. Essas áreas são prioritárias para aplicação de práticas de conservação do solo e da água, visto que o uso intensivo de maquinários de grande porte contribui para a compactação e impermeabilização do solo, aumentando o escoamento superficial da água e a remoção das partículas do solo.

As culturas em sucessão de milho e feijão apresentaram uma perda média de solo de $0,10 \mathrm{Mg} \mathrm{ha}^{-1} \mathrm{ano}^{-1}$. Esses valores são reduzidos devido à aplicação da prática de plantio direto, que é caracterizada como uma técnica de conservação do solo que reduz a erosão hídrica pela permanência de culturas remanescentes, o que protege o solo e contribui com o aumento dos teores de matéria orgânica.

Nos povoamentos de eucalipto a perda de solo média foi de $3,77 \mathrm{Mg} \mathrm{ha}^{-1} \mathrm{ano}^{-1}$. Os valores estão acima da média da área, devido à ausência de manejo conservacionista nessas áreas, caracterizados como plantio morro a baixo, que confere maior velocidade ao escoamento superficial.

A mata nativa apresentou a menor média de perda de solo, calculada em $0,0087 \mathrm{Mg} \mathrm{ha}^{-1} \mathrm{ano}^{-1}$, com perda total de $0,60 \mathrm{Mg} a n o^{-1}$. No cultivo de café em nível, que corresponde a 54,31\% da área, a média das perdas de solo foi de 2,09 $\mathrm{Mg} \mathrm{ha}^{-1}$ ano $^{-1}$ e a perda total de 497,85 $\mathrm{Mg} \mathrm{ano}^{-1}$. A contribuição das áreas de cafezais na perda total de solo é de 76,67 \% sendo, portanto, as áreas que mais fornecem sedimentos ao interflúvio. 


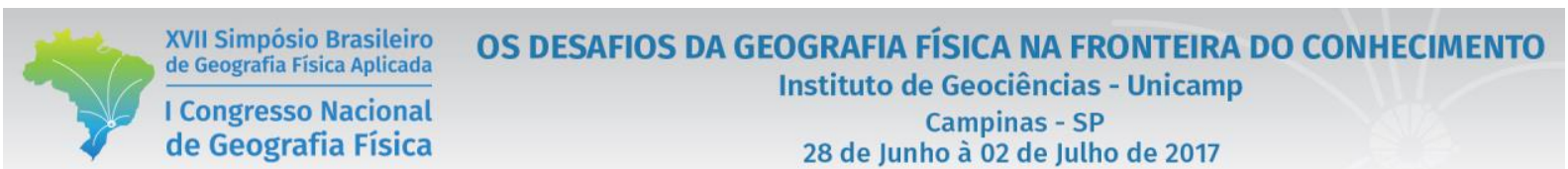

- TPS: Os valores da TPS foram de 5,19, 5,69 e 5,90 $\mathrm{Mg} \mathrm{ha}^{-1}$ ano-1 $^{-1}$, respectivamente para os solos LVd1, LVd2 e LVd3. O resultado da variável h foi $1.000 \mathrm{~mm}$, o valor de ponderação da variável $\mathrm{r}_{\mathrm{a}}$ foi 1 e a ponderação das variáveis $\mathrm{m}$ e $\mathrm{p}$ resultou no valor 0,7 , com a permeabilidade do solo classificada como lenta. A densidade dos solos, em $\mathrm{g} \mathrm{cm}^{-3}$, foi de 1,060, 1,163 e 1,205, respectivamente, para os solos LVd1, LVd2 e LVd3.

- Perdas de solo nos modelos RUSLE e EPM: Os valores das perdas de solo por unidade de área no modelo EPM foram transformados de $\mathrm{m}^{3} \mathrm{~km}^{-2} \mathrm{ano}^{-1}$ para $\mathrm{Mg} \mathrm{ha}^{-1} \mathrm{ano}^{-1}$, devido à utilização mais frequente da última no Brasil. Para a conversão das unidades de volume para massa foi utilizado à densidade média do solo de $1,150 \mathrm{~g} \mathrm{~cm}^{-3}$.

Os valores das perdas de solo nos modelos EPM (figura 3A) e RUSLE (figura 3B) estão descritos na tabela II.
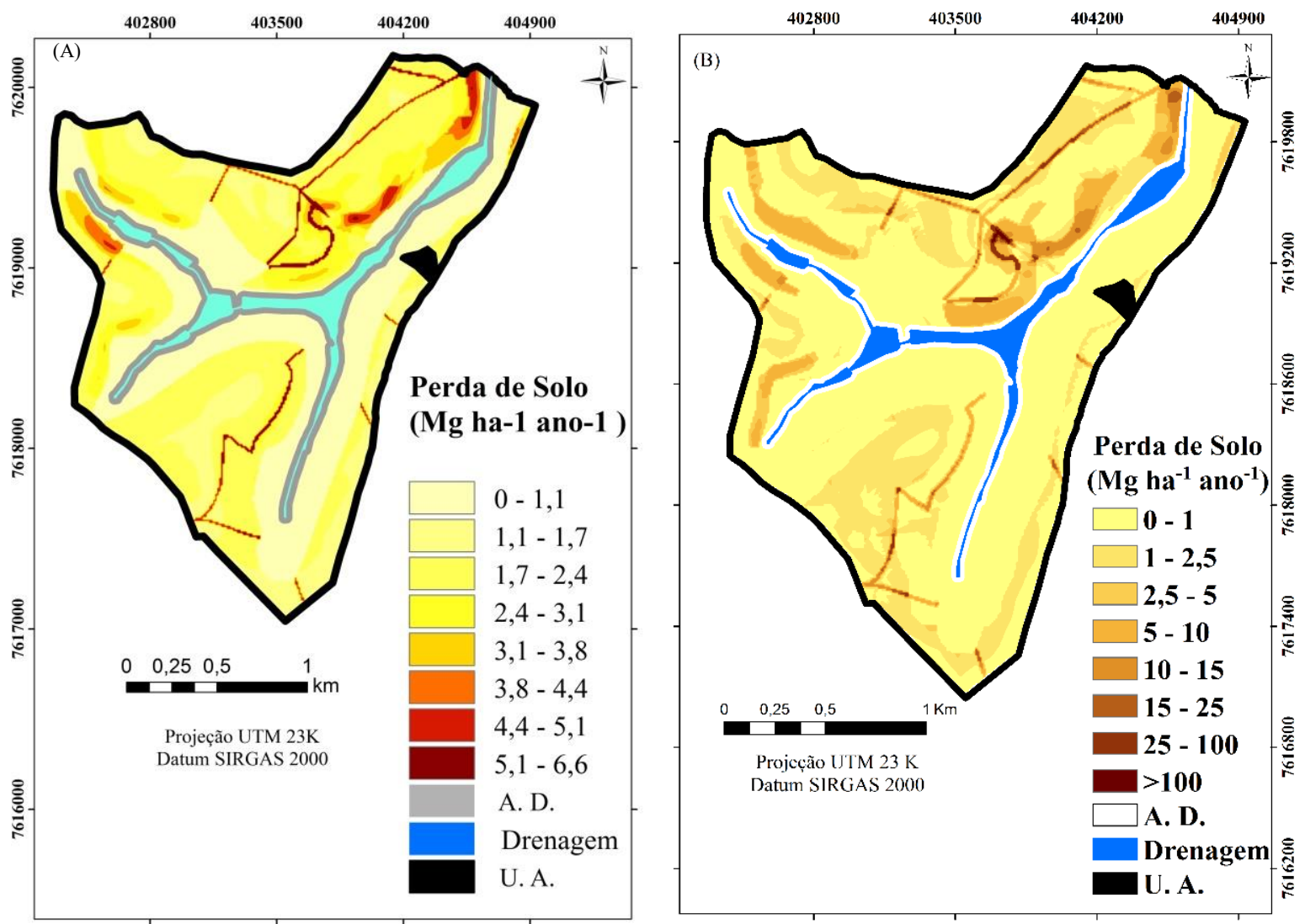

Figura 3: (A) Mapa das perdas de solo no modelo EPM e (B) no modelo RUSLE adaptado de Beskow et al. (2009).

Tabela II - Perdas de solo calculadas pelos modelos RUSLE e EPM.

\begin{tabular}{|c|c|c|c|c|c|}
\hline \multirow{2}{*}{$\begin{array}{l}\text { Classes de Uso } \\
\text { do Solo }\end{array}$} & \multirow[b]{2}{*}{ Área } & \multicolumn{2}{|c|}{ Perda média de solo } & Perda total de solo & Contribuição nas perdas \\
\hline & & RUSLE & EPM & RUSLE $\quad$ EPM & de solo $(\%)$ \\
\hline
\end{tabular}




$\begin{gathered}\text { XVII Simpósio Brasileiro } \\ \text { de Geografia Fisica Aplicada }\end{gathered}$
$\begin{aligned} & \text { I Congresso Nacional } \\ & \text { de Geografia Fisica }\end{aligned}$

RUSLE: Equação Universal da Perda de Solo Revisada. EPM: Método de Erosão Potencial.

A distribuição das perdas de solo na sub-bacia, em ambos os modelos, apresenta essencialmente áreas com erosão suave. Todavia, apresenta áreas prioritárias para a implantação de manejos conservacionistas que amenizem os efeitos deletérios da erosão. No modelo RUSLE cerca de $7 \%$ da área possui perdas de solo acima do limite da TPS, localizadas nos povoamentos de eucalipto e nos carreadores com solo exposto. No modelo EPM, tais áreas apresentam uma taxa de erosão máxima de $5,50 \mathrm{Mg} \mathrm{ha}^{-1}$ ano $^{-1}$, ligeiramente abaixo do limite da TPS. Logo, as mesmas devem ser objeto de monitoramento e mitigação das taxas de erosão.

\section{Conclusão}

As estimativas de perdas de solo no modelo EPM variaram entre 0,0087 e 5,50 $\mathrm{Mg} \mathrm{ha}^{-1} \mathrm{ano}^{-1}$, e no modelo RUSLE entre 0,04 e $18,64 \mathrm{Mg} \mathrm{ha}^{-1} \mathrm{ano}^{-1}$.

A perda média de solo no modelo EPM foi de 1,46 $\mathrm{Mg} \mathrm{ha}^{-1}$ ano $^{-1}$ e no modelo RUSLE de 1,52 $\mathrm{Mg} \mathrm{ha}^{-1}$ ano $^{-1}$, a baixo do limite mínimo da tolerância de perda de solo, que variou entre 5,19 e 5,90 Mg ha-1 ano ${ }^{-1}$.

No modelo RUSLE as áreas sob cultivo de eucalipto possui perda de solo de $6,05 \mathrm{Mg} \mathrm{ha}^{-1} \mathrm{ano}^{-1}$, enquanto no modelo EPM e carreadores de café, com perda de 18,64 $\mathrm{Mg} \mathrm{ha}^{-1}$ ano $^{-1}$, estão acima do limite mínimo da tolerância de perda de solo

Nos dois modelos estudados, as maiores perdas de solo estão associadas aos povoamentos de eucalipto e solos expostos. Todavia, apesar do modelo EPM não apresentar área com taxa de erosão acima do limite da tolerância de perda de solo, ambos os modelos indicam tais áreas como prioritárias para a mitigação das taxas de erosão.

\section{Agradecimentos}

À Ipanema Agrícola S.A. (Ipanema Coffees), pelo suporte logístico. 


\section{Referencial Bibliográfico}

AQUINO, R. F. et al. Spatial variability of the rainfall erosivity in Southern region of Minas Gerais State, Brazil. Ciência e Agrotecnologia. 36: 533-542, 2012.

AYER, J. E. B. et al. Erosão hídrica em Latossolos Vermelhos Distróficos. Pesquisa Agropecuária Tropical. 45: $180-191,2015$.

BERTOL, I.; ALMEIDA, J.A. Tolerância de perda de solo por erosão para os principais solos do estado de Santa Catarina. Revista Brasileira de Ciências do Solo, Viçosa, v. 24, n. 3, p. 657-668, 2000.

BERTONI, J.; LOMBARDI NETO, F. Conservação do solo. São Paulo, 8 ed. Ícone, p. 360, 2012.

BESKOW, S. et al. Soil erosion prediction in the Grande River Basin, Brazil, using distributed modeling. Catena, 79: 49-59, 2009.

BRASIL. Código Florestal. Lei n 12.651 de 25 de maio de 2012. [internet]. Brasília, DF: Congresso Nacional; 2012 [acesso em 15 mai 2015]. Disponível em: http://www.planalto.gov.br/ccivil_03/_Ato2011-

2014/2012/Lei/L12727.htm.

BRASIL. Ministério da Agricultura. Serviço Nacional De Conservação dos Solos. Levantamento de reconhecimento dos solos da região sob influência do reservatório de Furnas. Rio de Janeiro: Boletim Técnico, n.13, 1962.

CÂNDIDO, B. M. et al. Erosão Hídrica Pós-Plantio em Florestas de Eucalipto na Bacia do Rio Paraná, no Leste do Mato Grosso do Sul. Revista Brasileira de Ciências do Solo, Viçosa, v. 38, n. 5, p. 1565-1575, 2014.

CARVALHO R. et al. Erosão Hídrica em Latossolo Vermelho sob Diversos Sistemas de Manejo do Cafeeiro no Sul de Minas Gerais. Ciência e Agrotecnologia. 31: 1679-1687, 2007.

EMBRAPA, Empresa Brasileira de Pesquisa Agropecuária. Centro Nacional de Pesquisas de Solos. Manual de métodos de análise de solos. 2nd ed. Rio de Janeiro, 2011.

EMBRAPA, Empresa Brasileira de Pesquisa Agropecuária - EMBRAPA. Centro Nacional de Pesquisas de Solos. Sistema Brasileiro de Classificação de Solos. 3rd ed. Rio de Janeiro, 2013.

GALINDO, IC de L.; MARGOLIS, E. Tolerância de perdas por erosão para solos do Estado de Pernambuco. Revista Brasileira de Ciências do Solo, v. 13, n. 1, p. 95-100, 1989.

GAVRILOVIC, Z. The use of empirical method (erosion potential method) for calculating sediment production and transportation in unstudied or torrential streams. In: WHITE, W. R. (ed.), International Conference on River Regime, Chichester, p. 411-422, 1988.

HASUI, Y. A grande colisão pré-cambriana do Sudeste Brasileiro e a estruturação regional. Geociências, São Paulo, v.29, n.2, p. 141-169, 2010.

IBGE, Instituto Brasileiro de Geografia e Estatística. Carta Topográfica do Município de Alfenas (FOLHA SF 231-1-3). 1st ed. Rio de Janeiro, 1970.

IBGE, Instituto Brasileiro de Geografia e Estatística. Manual técnico de pedologia. 3rd ed. Rio de Janeiro, 2015.

MC BRATNEY, A. B.; MENDONÇA, S. M. L.; MINASNY B. On digital soil mapping. Geoderma. p. 117: 3-52. 2003.

MITASOVA H. M. et al. Terrain modelling and soil erosion: applications for Ft. Hood report for USA CERL [internet]. [acesso em 17 mai 2016]. Disponível em: <http://shagit.meas.ncsu.edu/ helena/gmslab/reports/cer101 /finalreport/report01/default.htm>. Champaign: University of Illinois, 2001.

OLIVETTI, D. et al. Spatial and Temporal Modeling of Water Erosion in Dystrophic Red Latosol (Oxisol) used for Farming and Cattle Raising Activities in a Sub-Basin in the South of Minas Gerais. Ciência e Agrotecnologia, 39: 58-67. 2015. 
PANAGOS, P.; BORRELLI, P.; MEUSBURGER, K. A New European Slope Length and Steepness Factor (LSFactor) for Modeling Soil Erosion by Water. Geosciences. 5:117-126, 2015.

PANAGOS, P. et al. Estimating the soil erosion cover-management factor at the European Scale. European Commission, Joint Research Centre, Institute for Environment and Sustainability, Environmental Geosciences, Land Use Police 48 University of Basel, Switzerland, p. 38-50, 2015.

PIMENTEL, D. et al. Environmental and Economic Costs of Soil Erosion and Conservation Benefits. SCIENCE, vol. 267, p. 1117-1123, 1995.

RENARD, K. G. et al. Predicting soil erosion by water: a guide to conservation planning with the Revised Universal Soil Loss Equation (RUSLE). United States Department of Agriculture - EUA. Agriculture Handbook. n. 703, 384 p. 1997.

ROOSE, E. J. Application of the Universal Soil Loss Equation of Wischmeier and Smith in West Africa. In: Greenland DJ, Lal R, editores. Soil conservation and management in the humid tropics. 1st ed. Chichester: John Wiley \& Sons. p. 177-187. 1977.

SANTOS R. D. et al. Manual de descrição e coleta de solos no campo.5th ed. Viçosa, Sociedade Brasileira de Ciência do Solo - Sbcs, Empresa Brasileira de Pesquisa Agropecuária - Embrapa, Centro Nacional de Pesquisa de Solos - Cnps, 2005.

SILVA, S.A. et al. Análise espacial da erosão hídrica em um latossolo vermelho amarelo sob cultivo de café conilon. Revista Ciência Agronômica. 38: 335-342, 2008.

SILVA M. L. N. et al. Proposição de modelos para estimativa da erodibilidade de Latossolos brasileiros. Pesquisa Agropecuária Brasileira. 34: 2287-2298, 1999.

SPALEVIC, V. Impact of land use on runoff and soil erosion in Polimlje. Doctoral thesis, Faculty of Agriculture of the University of Belgrade, Serbia, 260p. 2011.

SPAROVEK, G.; VAN LIER, Q. J.; DOURADO NETO, D. Computer assisted Köppen climate classification: a case study for Brazil. International Journal of Climatology. 27: 257-266, 2007.

ZHANG, R. Determination of soil sorptivity and hydraulic conductivity from the disk infiltrometer. Soil Science Society of America Journal, 61: 1024-1030. 1997 